\title{
Influence of fine sediment on the fluidity of debris flows
}

\author{
Norifumi HOTTA ${ }^{*}$, Takahiro KANEKO², Tomoyuki IWATA ${ }^{3}$, Haruo NISHIMOTO ${ }^{1}$ \\ 1 Faculty of Life and Environmental Sciences, University of Tsukuba, 3058577 \\ Ibaraki, Japan \\ 2 Faculty of Agriculture, The University of Tokyo, 1138657 Tokyo, Japan \\ 3 Graduate School of Agricultural and Life Sciences, The University of Tokyo, \\ 1138657 Tokyo, Japan \\ *Corresponding Author, e-mail: hotta.norifumi.ge@u.tsukuba.ac.jp
}

\begin{abstract}
Debris flows include a great diversity of grain sizes resulting in inherent features such as inverse grading, particle size segregation, and liquefaction of fine sediment. The liquefaction of fine sediment affects the fluidity of debris flows, although the behavior and influence of fine sediment in debris flows have not been examined sufficiently. This study used flume tests to detect the effect of fine sediment on the fluidity of laboratory debris flows consisting of particles with various diameters. From the experiments, the greatest sediment concentration and flow depth were observed in the debris flows mixed with fine sediment indicating increased flow resistance. The experimental friction coefficient was then compared with the theoretical friction coefficient derived by substituting the experimental values into the constitutive equations for debris flow. The theoretical friction coefficient was obtained from two models with different fine-sediment treatments: assuming that all of the fine sediments were solid particles or that the particles consisted of a fluid phase involving pore water liquefaction. From the comparison of the friction coefficients, a fully liquefaction state was detected for the fine particle mixture. When the mixing ratio and particle size of the fine sediment were different, some other cases were considered to be in a partially liquefied transition state. These results imply that the liquefaction of fine sediment in debris flows was induced not only by the geometric conditions such as particle sizes, but also by the flow conditions.
\end{abstract}

\section{Keywords:}

debris flow, fine sediment, friction coefficient, liquefaction, open channel, Reynolds stress 


\section{Introduction}

The basic equations for debris flows have been derived from simple modeling of the laminar motion of sediment particles (Takahashi, 1977; Tsubaki et al., 1982; Egashira et al., 1997). These equations have been validated experimentally by comparing the theoretical and experimental velocity distributions (Takahashi, 1977; Egashira et al., 1989; Itoh and Egashira, 1999) and the flow resistance (Arattano and Franzi, 2004; Hotta and Miyamoto, 2008). Debris flows have been classified into "boulder debris flows" or "stony debris flows", and the interparticle stresses induced by particle-to-particle collisions and particle friction in the flow have been considered. In many cases, the particle size is assumed to be uniform and the mean diameter or $d_{50}$ is used as a representative sediment particle size when applying these models to actual debris flows.

However, in-situ debris flows often include a great variety of grain sizes, including a relatively high portion of fine sediment. When debris flows contain a large amount of fine sediment, this sediment can affect the fluidity. Rickenmann (1991) and Takahashi and Kobayashi (1993) investigated the influence of fluid viscosity of clay suspensions on the fluidity of debris flows that consisted of clay and coarse particles, and found that the viscous coefficient of the pore fluid altered the total shear stress. In addition, Egashira et al. (1989, 1997) modeled a component of the shear stresses in the pore fluid of boulder debris flows as the Reynolds stress. Although sediment particles in the boulder debris flows, themselves, move laminar motion, the pore fluid should be turbulent because of the strong shear induced by the sediment particles. Hotta and Miyamoto (2008) pointed out that the friction coefficient in turbulent sediment flows was comparable with that of clear water; thus, the fine sediment contributes to the fluidity, even when the mass density of the interstitial fluid is increased without increasing the viscosity. Using the same concept, Nishiguchi et al. (2011) modeled debris flows with mixed grain sizes and large flow depths in which fine sediment was involved in the interstitial water; by doing so, they were better able to predict the run-out of large debris flows.

We conducted flume tests to detect the effect of fine sediment on the fluidity or the difference, due to different particle sizes of fine sediment in debris flows consisting of particles with mixed diameters. The experimental and theoretical flow resistances were then compared with those derived from experimental results to investigate the behavior of fine sediment.

\section{Experiment}

The variable slope channel of the Civil Engineering Research Laboratory (904-1 Tohigashi, Tsukuba, Ibaraki, Japan) was used for the experiments (Figure 1). The channel is $10 \mathrm{~m}$ long and $30 \mathrm{~cm}$ wide, with glazed sides. In these experiments, the width of the channel was reduced to $10 \mathrm{~cm}$ and the bottom of the lower stream of the channel $(4.5 \mathrm{~m})$ was raised as high as $10 \mathrm{~cm}$. Sediment particles, $2.9 \mathrm{~mm}$ in diameter, were glued in the lower stream to provide bed roughness. An ultrasonic sensor (E4PA-LS50-M1, Omron, Kyoto, Japan) was installed $1 \mathrm{~m}$ above the lower end to measure the temporal change in the flow surface level at a sampling rate of $20 \mathrm{~Hz}$.

The upper stream of the channel was filled with particles to a depth of about $10 \mathrm{~cm}$. A steady flow of water was supplied from the upper end to generate a debris flow by eroding 
sediment deposits in the upper section of the channel. The debris-flow sample was captured using a sampler at the downstream end, and the sampling time was recorded. The unit width flux $Q$ and sediment flux concentration were obtained using the debris-flow sample. The debris-flow samples were obtained in the steady-state section, which could be verified by referring to the time series of the surface level data measured by an ultrasonic displacement sensor, as Hotta (2012) demonstrated using the same experimental setup. The average flow depth $h$ was also obtained for the steady-state section, and the vertical (and cross-sectional) average flow velocity $u_{m}$ was determined from the following relationship:

$$
Q=h u_{m}
$$

Silica sands of five particle sizes were used in the experiments. Table 1 shows the particle sizes (mean diameter for 0.84-2.9 mm-sand and $d_{50}$ for 0.11 - and 0.23-mm sand) and the mixing ratio used in the experiments. The $0.84-2.9 \mathrm{~mm}$ sand was sieved to be as uniform as possible. The 2.9-mm sand was described as "large" particles. In addition to the two mixed diameters, tests were conducted using monogranular particles of the five sands. The mass density and interparticle friction angle of the sediment particles were 2.6 and $34^{\circ}$, respectively. The channel slope was set at $15^{\circ}$, and water was supplied from the upper end at about $3 \mathrm{Ls}^{-1}$.

\section{Analysis}

The friction coefficient $f$ was used to compare the experimental results and the predicted values to examine the influence of fine sediment on fluidity. Here, experimental $f$ was calculated using the following equation:

$$
f=\frac{2 g h \sin \theta}{u_{m}^{2}}
$$

where $g$ is the acceleration due to gravity, $h$ is the flow depth, $\theta$ is the channel slope, and $u_{m}$ is the mean velocity.

The theoretical $f$ for boulder debris flows over a rigid bed was obtained as follows. The energy dissipation $(\Phi)$ over a unit volume and time to achieve a steady debris flow is equal to the external energy supplied:

$$
\Phi=\rho_{m} g u \sin \theta
$$

where $\rho_{\mathrm{m}}$ is the mass density of the debris flow and $u$ is the velocity. Integrating Eq. (3) from the bed to the surface and substituting the result into Eq. (2) results in the following equations:

$$
\begin{aligned}
& \int_{0}^{h} \Phi d z=\int_{0}^{h} \rho_{m} g u \sin \theta d z=\rho_{m} g h u_{m} \sin \theta \\
& f=\frac{2 g h \sin \theta}{u_{m}{ }^{2}}=\frac{2}{\rho_{m}} \frac{1}{u_{m}{ }^{3}} \int_{0}^{h} \Phi d z
\end{aligned}
$$

Here, $\Phi$ can be rewritten as follows, based on the constitutive equations for debris flows proposed by Egashira et al. (1997):

$$
\Phi=K(c) d^{2}\left(\frac{\partial u}{\partial z}\right)^{3}
$$

where $d$ is the particle size, $c$ is the volumetric sediment concentration, and $K(c)$ is an equation 
expressed as a function of $c$. Refer to Hotta and Miyamoto (2008) for more detailed information on $K(c)$.

To use the friction coefficient in our analysis, we postulated that the mixture of sediment and water act as a single fluid, while sediment concentration in debris flow generally differs according to the flow depth. In this study, a constant value was assumed for $c$, and the velocity profile was assumed to be a typical velocity profile for boulder debris flows over a rigid bed (Egashira et al., 1989), which is also known as the velocity profile of a dilatant fluid (Takahashi, 1977) and can be expressed as

$$
\frac{\partial u}{\partial z}=\frac{5 u_{m}}{2 h}\left(1-\frac{z}{h}\right)^{1 / 2}
$$

Then, $f$ for debris flows can be obtained by substituting Eqs. (6) and (7) into Eq. (5) to obtain

$$
f=\frac{25}{2 \rho_{m}} K(c)\left(\frac{h}{d}\right)^{-2}
$$

Hotta and Miyamoto (2008) reported that the experimental and theoretical $f$ corresponded well for boulder debris flow in various flume tests, although the $f$ value should be obtained for steady-state, uniform sections of the debris flows.

In this study, two models were applied to calculate the theoretical friction coefficient for the debris flows with sand of two mixed diameters, as shown in Figure 2. Model I assumed that all of the fine (small) sediment acted as a solid phase; in this case, the sediment diameter was calculated as a mean value using the large and small particle diameters. Conversely, Model II assumed that all of the fine (small) sediment was involved in the interstitial water and acted as a fluid phase. In Model II, the sediment diameter was set to that of the large particles (2.9 $\mathrm{mm}$ ), the sediment concentration $c$ was derived using only the large-sediment volume, and the mass density of water $\rho$ was calculated including the fine sediment. Note that a transition between Models I and II is possible. In the transition state, fine sediment is partially involved in the pore fluid showing the intermediate value of the friction coefficient between Models I and II.

\section{Results and discussion}

\subsection{Experimental results}

Figure 3 shows the sediment concentrations in each experiment. The bars on the left in all figures indicate the value for experiments carried out with uniform 2.9-mm particles. The other three bars indicate the experiments carried out with mixing ratios of 1:4 and 1:1, and a uniform particle experiment with small particles. The concentrations were divided into concentrations of small and large particles, showing that the proportions were almost the same as the initial mixing ratios. In the cases with 1.3- and o.8-mm particles (Figure $3 \mathrm{a}, \mathrm{b}$ ), the total sediment concentrations were almost the same as in the cases with uniform particle sizes. However, the sediment concentration increased in the laboratory debris flows of mixed 0.2and o.1-mm particles (Figure 3c, d). For a mixing ratio of 1:4, the concentration of large (2.9-mm) particles was high enough to reach the concentration of debris flows with uniform large (2.9-mm) particles. 
Even when the fluxes of the laboratory debris flows were the same, the relationship between flow depth and velocity varied with the flow conditions. Figure 4 shows the flow depth for the experiments involving uniform particle sizes and mixed particles of two diameters. The bars on the left show the flow depth for the experiment with uniform 2.9-mm particles. The flow depth was greatest in the experiment with uniform 2.9-mm particles, as a consequence of the experiment with mixed o.2- and o.1-mm particles when the mixing ratio was 1:4.

\subsection{Comparison of the friction coefficients}

The modeled friction coefficients were compared with the experimental friction coefficients, as shown in Figure 5. For the experiments with uniform particle sizes (Figure 5a), both the experimental and theoretical friction coefficients were in good agreement for the experiments with 2.9-mm particles. The theoretical friction coefficients were smaller for smaller particles and were far from the experimental values for 0.23- and 0.11-mm particles. In those experiments, the flow conditions were considered turbulent (Hotta and Miyamoto, 2008); however, the theoretical friction coefficient was derived from the constitutive equations for boulder debris flows in which the flow is regarded as laminar, focusing on the sediment particle motion.

In the experiments using mixed particle sizes, both Models I and II could not sufficiently explain the experimental results. In Model I, the experimental and theoretical friction coefficients were in good agreement for the $0.84-\mathrm{mm}$ particles with a mixing ratio of 1:1 (Figure $5 \mathrm{~b}$ ) and for the $0.23-\mathrm{mm}$ particles with a mixing ratio of 1:4 (Figure 5c). In Model II, the experimental and theoretical friction coefficients were in good agreement for the 0.11-mm particles with a mixing ratio of 1:1 (Figure $5 \mathrm{~d}$ ), and for the 0.11 -and 0.23 -mm particles with a mixing ratio of 1:4 (Figure 5e). A comparison of the friction coefficients obtained from Models I and II can be summarized as follows. The cases using the $0.84-$ and 1.3-mm particles alone or the $0.23-\mathrm{mm}$ particles with a mixing ratio of 1:4 were well described by Model I: small particles were regarded as a solid phase. However, the friction coefficient of the $0.23-\mathrm{mm}$ particles did not differ significantly between Models I and II. Model II described well the case with the 0.11-mm particles with a mixing ratio of 1:4: the small particles acted as a fluid phase. The cases with 0.11 - and $0.23-\mathrm{mm}$ particles with a mixing ratio of 1:1 showed intermediate values or a better fit with Model II for the 0.11-mm particles. These cases suggest a transition from Model I to II; i.e., fine sediment in the debris flows existed in both solid and fluid phases, and there was a transition between the two phases depending on the flow conditions.

This idea was supported by the experimental results. As the sediment concentration increased in cases with smaller particles ( 0.11 and $0.23 \mathrm{~mm}$; Figure 3c, d), geometric conditions such as the particle-diameter ratio allowed fine sediment to fill the pore spaces of the coarse grains. When the mixing ratio was 1:4, the flow depth in cases with mixed particles increased to approximate the flow depth in the experiment with uniform 2.9-mm particles (Figure 4b). Assuming that different flow depths under the same conditions represent different flow resistances, the friction coefficient is well described by Model II only when all of the fine sediment is loaded in the interstitial space, such as with a mixing ratio of 1:4 (Figure 3c, d). In this situation, internal stresses due to particle-to-particle collisions and friction in the debris flow should mainly involve interactions among large particles. Such kinematic conditions can 
206 probably allow us to regard the behavior of fine sediment in the interstitial space of large

207 particles as liquefaction.

\section{Conclusion}

This study conducted flume tests to detect the effect of fine sediment on the fluidity of laboratory debris flows. The experiment used particles of two sizes: fine sediment consisting of $0.11^{-}, 0.23^{-}, 0.84^{-}$, or 1.3-mm particles and coarse grains fixed at a size of $2.9 \mathrm{~mm}$. From the experiments, the greatest sediment concentration and flow depth were observed in the debris flows with fine sediment containing 0.11- or $0.23-\mathrm{mm}$ particles, indicating increased flow resistance. The experimental and theoretical flow resistances were also derived from experimental results to investigate the behavior of fine sediment. Comparing the friction coefficients, complete liquefaction was observed in the experiment using $0.11-\mathrm{mm}$ particles with a mixing ratio of $1: 4$. The cases with $0.11-\mathrm{mm}$ particles with a mixing ratio of $1: 4$, and both 0.11- and $0.23-\mathrm{mm}$ particles with a mixing ratio of 1:1, were considered transition states. These results infer that the liquefaction of fine sediment in debris flows was induced not only by the geometric conditions, but also by the flow conditions such as internal stresses among sediment particles. That is, it is possible that "fine sediment" and "coarse grains" in debris flows of mixed particle sizes can be defined according to the kinematic conditions.

\section{Acknowledgments}

I sincerely thank Prof. Miyamoto (University of Tsukuba) for his comments on this study. I also thank Mr. Sugiura and Mr. Hasegawa (Civil Engineering Research Laboratory, Ibaraki, Japan) for their help with the experiments. This research was partially supported by Grant-in-Aid for Scientific Research 22780140, 2010, from the Ministry of Education, Science, Sports, and Culture, of Japan. 


\section{References}

Arattano M, Franzi L (2004) Analysis of different water-sediment flow processes in a mountain torrent. Natural Hazard and Earth System Sciences 4: 783-791.

Egashira S, Ashida K, Yajima H, Takahama J (1989) Constitutive equations of debris flow. Annals of the Disaster Prevention Research Institute, Kyoto University 32 (B-2): 487-501. (in Japanese with English summary)

Egashira S, Miyamoto K, Itoh T (1997) Constitutive equations of debris flow and their applicability. In: Proceedings of the 1st International Conference on Debris-Flow Hazards Mitigation, San Francisco, California, US, 7-9 August 1997. pp 340-349.

Hotta N, Miyamoto K (2008) Phase classification of laboratory debris flows over a rigid bed based on the relative flow depth and friction coefficients. International Journal of Erosion Control Engineering 1 (2): 54-61.

Hotta N (2012) Basal interstitial water pressure in laboratory debris flows over a rigid bed in an open channel. Natural Hazards and Earth System Sciences 12: 2499-2505.

Itoh T, Egashira S (1999) Comparative study of constitutive equations for debris flows. Journal of Hydroscience and Hydraulic Engineering 17 (1): 59-71.

Nishiguchi Y, Uchida T, Tamura K, Satofuka Y (2011) Prediction of run-out process for a debris flow triggered by a deep rapid landslide. In: Debris-Flow Hazards Mitigation: Mechanics, Prediction and Assessment, Genevois, R., Hamilton, D. L., Prestininzi, A. eds., Casa Editrice Universita La Sapienza, Roma. pp 477-485.

Rickenmann D (1991) Hyperconcentrated flow and sediment transport at steep slopes. Journal of Hydraulic Engineering 117 (11): 1419-1439.

Takahashi T (1977) A mechanism of occurrence of mud-debris flows and their characteristics in motion. Annals of the Disaster Prevention Research Institute, Kyoto University 20 (B-2): 405-435. (in Japanese with English summary)

Takahashi T, Kobayashi K (1993) Mechanics of the viscous type debris flow. Annals of the Disaster Prevention Research Institute, Kyoto University 36 (B-2): 433-449. (in Japanese with English summary)

Tsubaki T, Hashimoto H, Suetsugi T (1982) Grain stresses and flow properties of debris flows. In: Proceedings of the Japan Society of Civil Engineers 317: 79-91. (in Japanese) 
Table 1 Particle sizes and mixing ratios used in the experiments. Large and small particles were mixed for experiments involving mixed diameters.

\begin{tabular}{ccc} 
Small $(\mathrm{mm})$ & Large $(\mathrm{mm})$ & Mixing ratio \\
\hline 1.3 & & $1: 1$ \\
0.84 & 2.9 & and \\
0.23 & & $1: 4$ \\
0.11 & & \\
\hline
\end{tabular}


Figure legends

Figure 1 Experimental setup.

Figure 2 Schematic diagrams of Models I and II. When the fine sediment is involved (liquefied) in the pore fluid, representative sediment particle size, sediment concentration, and density of the pore fluid change.

Figure 3 Sediment concentrations for the (a) 1.3-, (b) 0.84-, (c) 0.23-, and (d) 0.11-mm sediment particle experiments. Monogranular experiments and mixing ratios of 1:4 and 1:1 are shown in each column. The bars on the left in all figures indicate the sediment concentration for the uniform 2.9-mm particle experiments.

Figure 4 Flow depth for (a) uniform particle sizes and mixing ratios of (b) 1:4 and (c) 1:1. The bars to the left in (b) and (c) indicate the flow depth for the uniform 2.9-mm particle experiments as a reference.

Figure 5 Relationship between the experimental and theoretical friction coefficients for (a) uniform particle sizes (Model I), mixing ratios of (b) 1:1 and (c) 1:4 with Model I, and mixing ratios of (d) 1:1 and (e) 1:4 with Model II. 


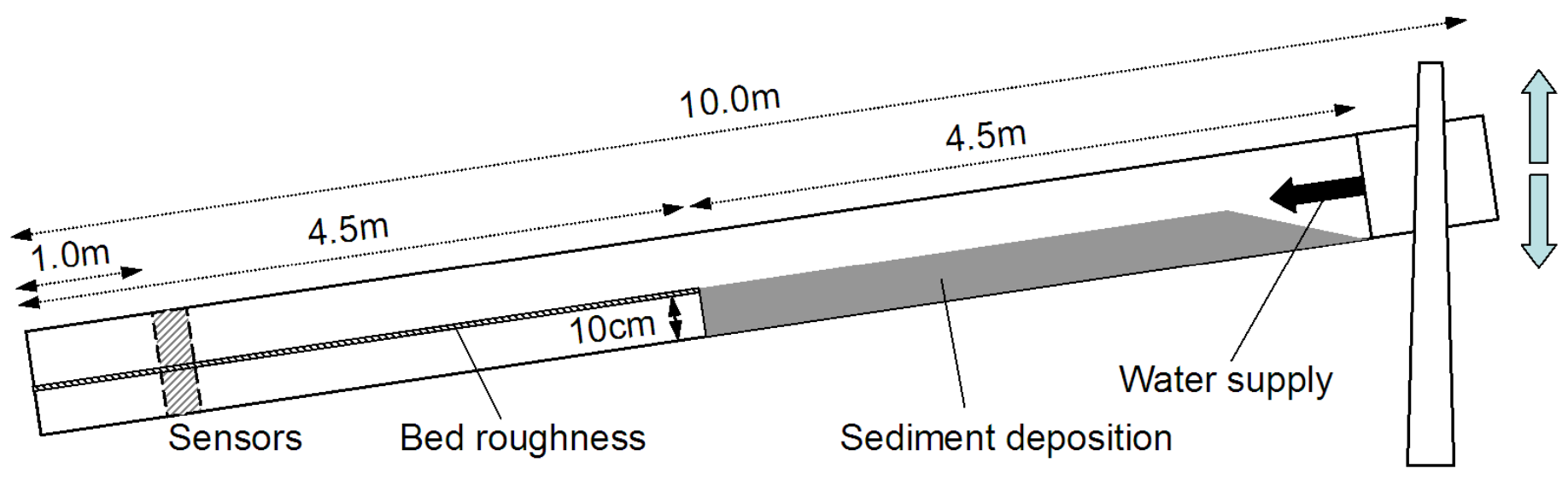

Figure 1 Experimental setup. 


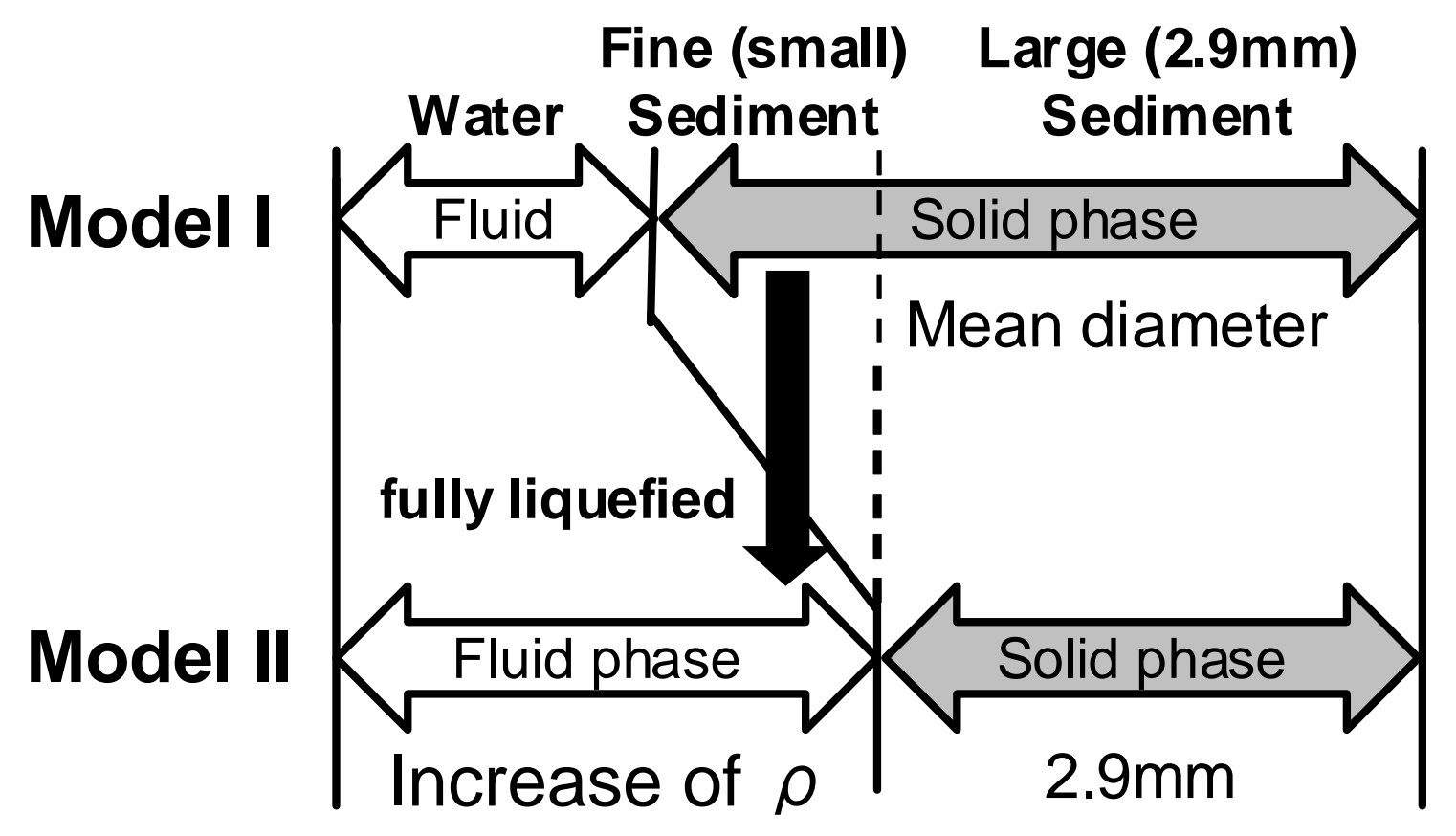

Figure 2 Schematic diagrams of Models I and II. When the fine sediment is involved (liquefied) in the pore fluid, representative sediment particle size, sediment concentration, and density of the pore fluid change. 

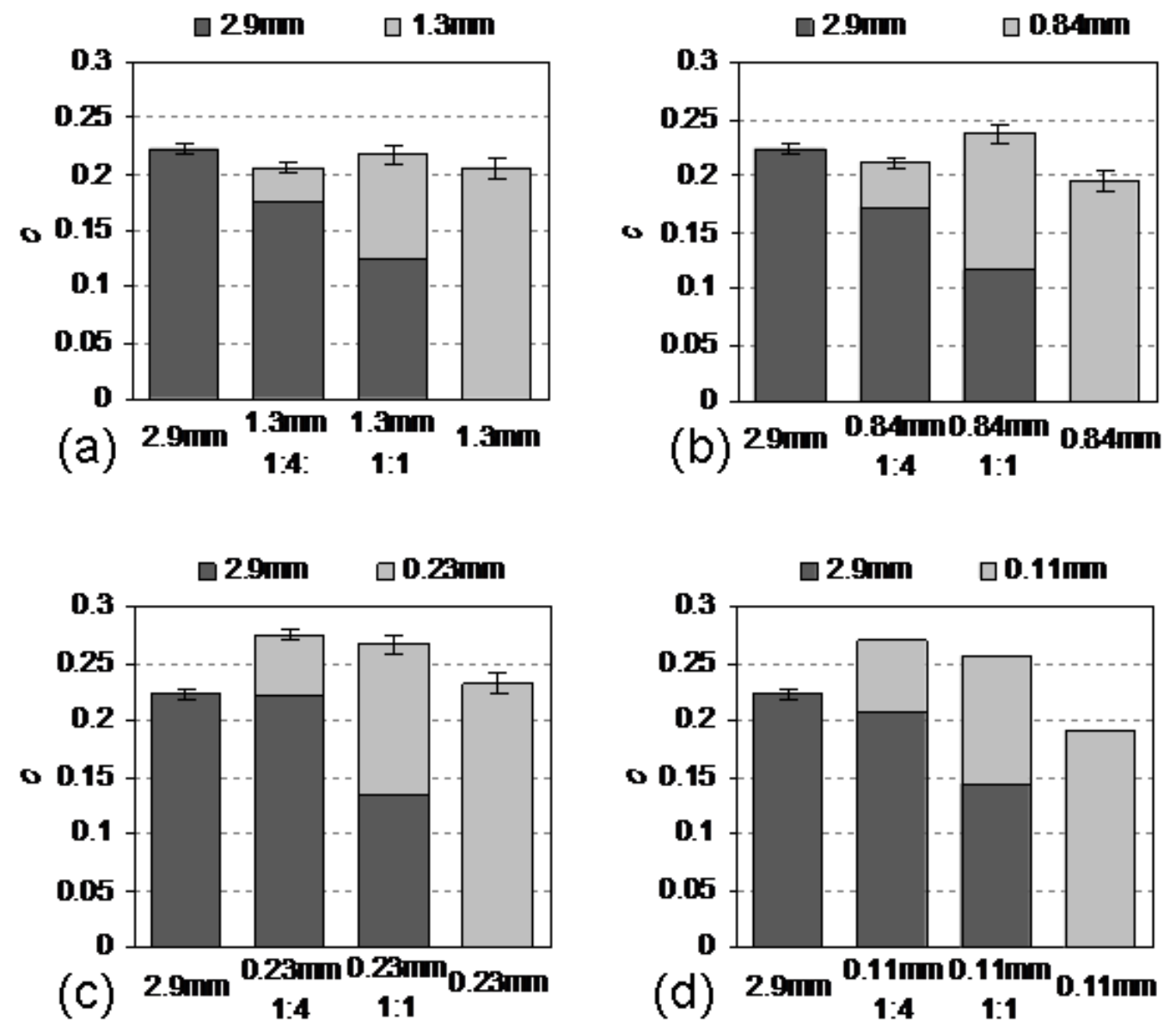

Figure 3 Sediment concentrations for the (a) 1.3-, (b) 0.84-, (c) 0.23-, and (d) 0.11-mm sediment particle experiments.

Monogranular experiments and mixing ratios of 1:4 and 1:1 are shown in each column. The bars on the left in all figures indicate the sediment concentration for the uniform 2.9-mm particle experiments. 


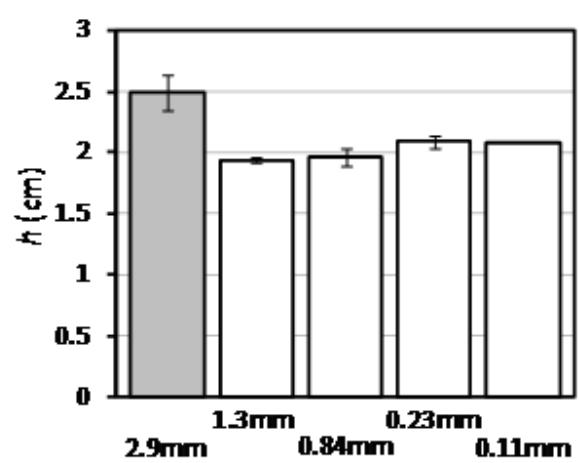

(a) Uniform particle sizes

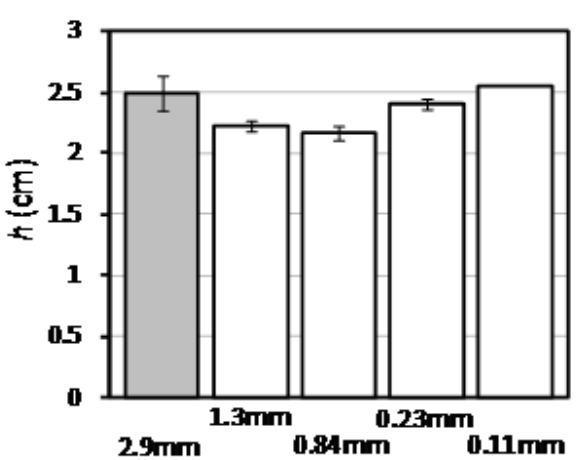

(b) Mixing ratio of 1:4

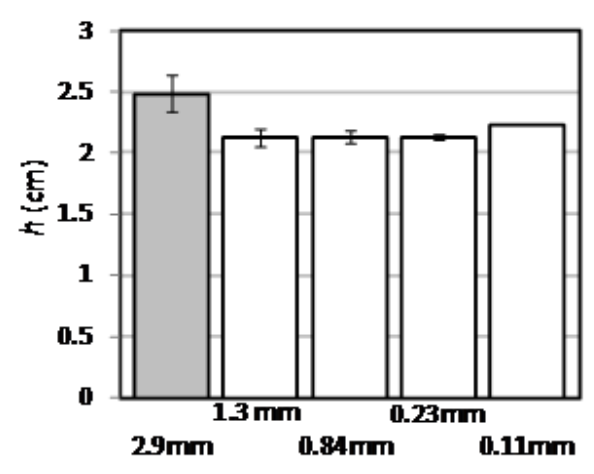

(c) Mixing ratio of $1: 1$

Figure 4 Flow depth for (a) uniform particle sizes and mixing ratios of (b) 1:4 and (c) 1:1. The bars to the left in (b) and (c) indicate the flow depth for the uniform 2.9-mm particle experiments as a reference. 


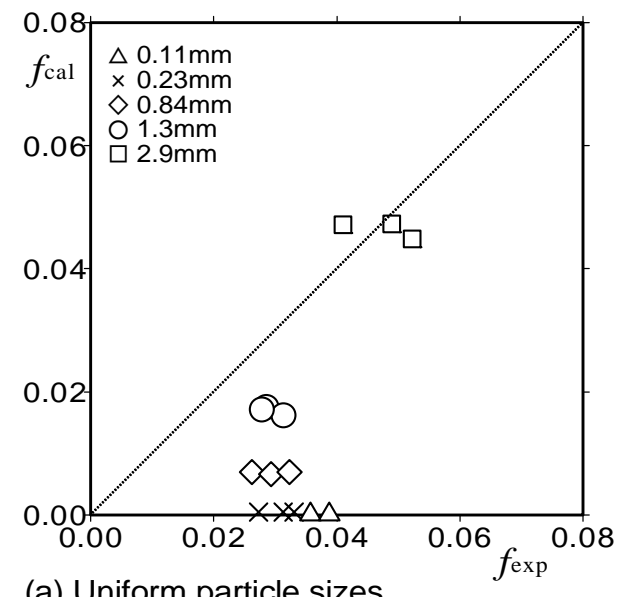

(a) Uniform particle sizes

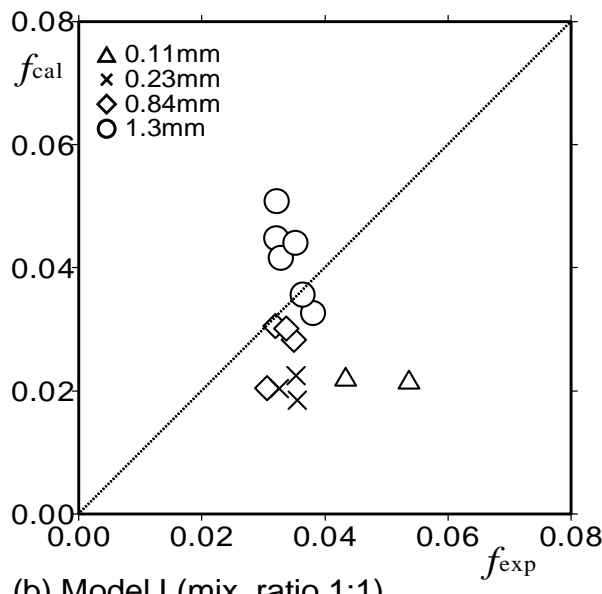

(b) Model I (mix. ratio 1:1)

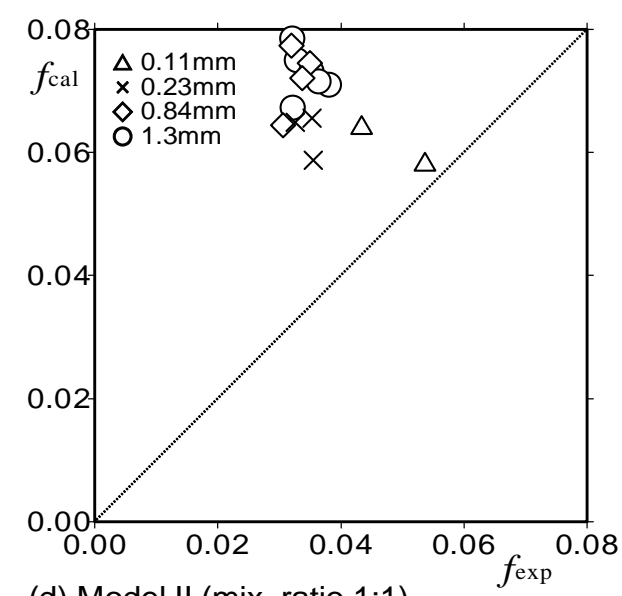

(d) Model II (mix. ratio 1:1)

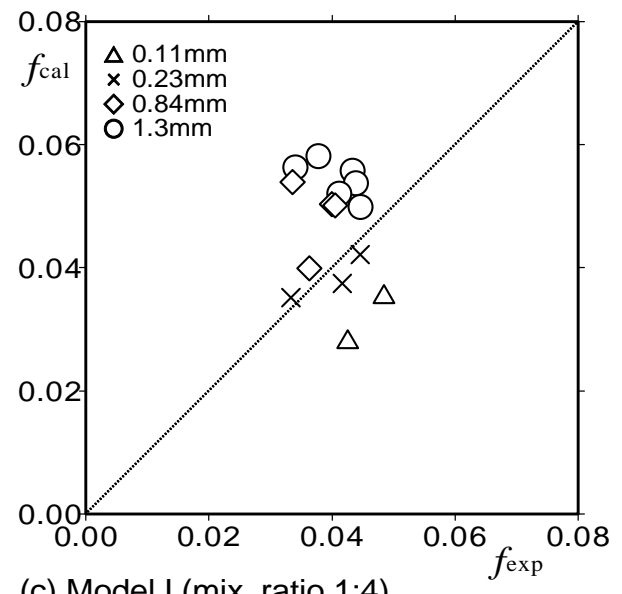

(c) Model I (mix. ratio 1:4)

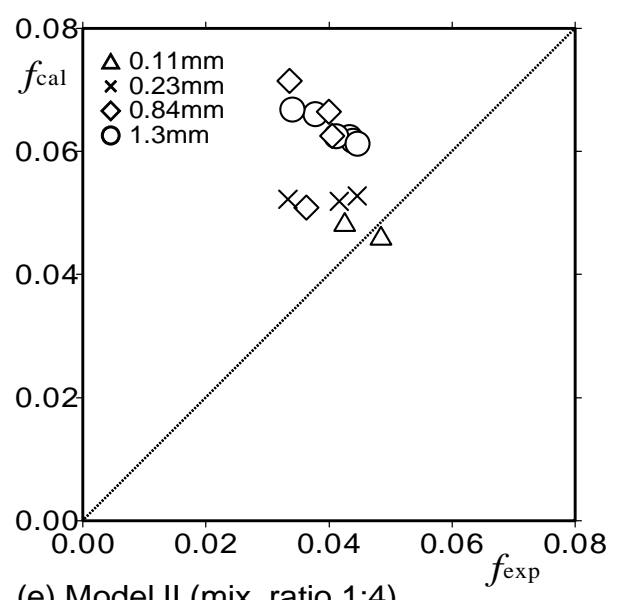

(e) Model II (mix. ratio 1:4)

Figure 5 Relationship between the experimental and theoretical friction coefficients for (a) uniform particle sizes (Model I), mixing ratios of (b) 1:1 and (c) 1:4 with Model I, and mixing ratios of (d) 1:1 and (e) 1:4 with Model II. 